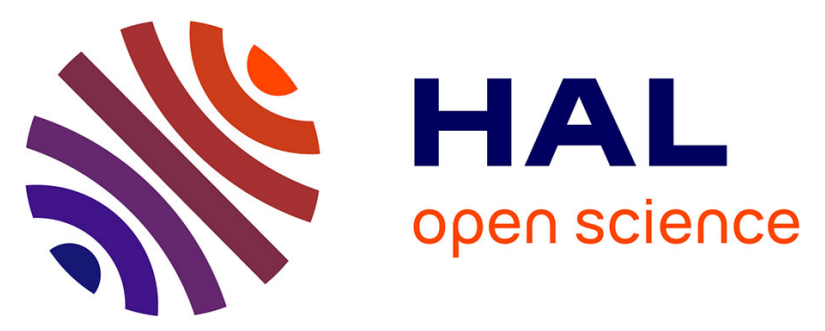

\title{
Reverse electrochemical etching method for fabricating ultra-sharp platinum/iridium tips for combined scanning tunneling microscope/ atomic force microscope based on a quartz tuning fork
}

José A. Morán-Meza, Jérôme Polesel-Maris, Christophe Lubin, François Thoyer, Ali Makky, Abdelkarim Ouerghi, Jacques Cousty

\section{To cite this version:}

José A. Morán-Meza, Jérôme Polesel-Maris, Christophe Lubin, François Thoyer, Ali Makky, et al.. Reverse electrochemical etching method for fabricating ultra-sharp platinum/iridium tips for combined scanning tunneling microscope/ atomic force microscope based on a quartz tuning fork. Current Applied Physics, 2015, 15, pp.1015-1021. 10.1016/j.cap.2015.05.015 . cea-01273519

HAL Id: cea-01273519

https://hal-cea.archives-ouvertes.fr/cea-01273519

Submitted on 12 Feb 2016

HAL is a multi-disciplinary open access archive for the deposit and dissemination of scientific research documents, whether they are published or not. The documents may come from teaching and research institutions in France or abroad, or from public or private research centers.
L'archive ouverte pluridisciplinaire HAL, est destinée au dépôt et à la diffusion de documents scientifiques de niveau recherche, publiés ou non, émanant des établissements d'enseignement et de recherche français ou étrangers, des laboratoires publics ou privés. 


\title{
Reverse electrochemical etching method for fabricating ultra-sharp platinum/iridium tips for combined scanning tunneling microscope/ atomic force microscope based on a quartz tuning fork
}

\author{
J.A. Morán Meza ${ }^{\text {a, b, * }, ~ J . ~ P o l e s e l-M a r i s ~}{ }^{\text {a, } 1}$, C. Lubin ${ }^{\text {a }}$, F. Thoyer ${ }^{\text {a }}$, A. Makky ${ }^{\mathrm{a}, 2}$, A. Ouerghi ${ }^{\text {, }}$ \\ J. Cousty ${ }^{\mathrm{a}}$ \\ a Service de Physique de l'Etat Condensé, DSM/IRAMIS/SPEC, CNRS UMR 3680, CEA Saclay, 91191 Gif sur Yvette Cedex, France \\ b Grupo de Materiales Nanoestructurados, Facultad de Ciencias, Universidad Nacional de Ingeniería, Av. Túpac Amaru 210, Lima 25, Peru

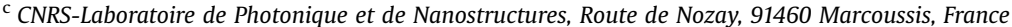

\section{A R T I C L E I N F O}

\section{Article history:}

Received 11 February 2015

Received in revised form 13 May 2015

Accepted 27 May 2015

Available online 17 June 2015

\section{Keywords:}

Tip etching

AFM

STM

Fowler-Nordheim plot

Epitaxial graphene

\begin{abstract}
A B S T R A C T
Sharp Pt/Ir tips have been reproducibly etched by an electrochemical process using an inverse geometry of an electrochemical cell and a dedicated electronic device which allows us to control the applied voltages waveform and the intensity of the etching current. Conductive tips with a radius smaller than $10 \mathrm{~nm}$ were routinely produced as shown by field emission measurements through Fowler-Nordheim plots. These etched tips were then fixed on a quartz tuning fork force sensor working in a qPlus configuration to check their performances for both scanning tunneling microscopy (STM) and atomic force microscopy (AFM) imaging. Their sharpness and conductivity are evidenced by the resolution achieved in STM and AFM images obtained of epitaxial graphene on $6 \mathrm{H}-\mathrm{SiC}(0001)$ surface. The structure of an epitaxial graphene layer thermally grown on the $6 \mathrm{H}-\mathrm{SiC}(0001)(6 \sqrt{3} \times 6 \sqrt{3}) \mathrm{R}^{\circ} 0^{\circ}$ reconstructed surface, was successfully imaged at room temperature with STM, dynamic STM and by frequency modulated AFM.
\end{abstract}

(c) 2015 Elsevier B.V. All rights reserved.

\section{Introduction}

Recently, several high-resolution atomic force microscopy (AFM) studies of surface structures were performed with selfsensing piezoelectric scanning probes working in ultrahigh vacuum environment [1-4] or in liquid [5,6]. The probe often consists of a sharp metallic tip attached to a quartz tuning fork. In the qPlus configuration [1], one prong of the tuning fork is fixed while the tip is fixed on the end of the oscillating free prong. With a spring constant comprised between 1 and $10 \mathrm{kN} / \mathrm{m}$, this stiff AFM probe can also produce scanning tunneling microscopy (STM) images

\footnotetext{
* Corresponding author. Postal address: 31-139, Lima, Peru.

E-mail addresses: jmoranm@uni.edu.pe (J.A.M. Meza), jpoleselmaris@ groupeseb.com (J. Polesel-Maris), christophe.lubin@cea.fr (C. Lubin), francois. thoyer@cea.fr (F. Thoyer), ali.makky@u-psud.fr (A. Makky), abdelkarim.ouerghi@ lpn.cnrs.fr (A. Ouerghi), jacques.cousty@cea.fr (J. Cousty).

1 Present address: Tefal SAS, 15 Avenue des Alpes, Zae Rumilly Est - BP 89, F 74156 Rumilly Cedex, France.

2 Present address: Institut Galien Paris-Sud, CNRS UMR 8612, University of Paris-Sud, F-92196, Châtenay-Malabry Cedex, France.
}

since a short distance of a few atomic diameters can be established between the oscillating metallic tip and the surface. In that oscillating tip STM mode, hereafter called dynamic STM, the Z distance regulation is driven by the mean value of the tunneling current circulating between the tip and the conductive surface while the corresponding resonance frequency shift variations sensitive to the surface force gradient can be recorded. Such a combined STM/AFM system appears very attractive since different properties of the surface can be simultaneously investigated. In this context, the tip sharpness is the key for obtaining high resolution images.

The production of sharp metallic tips was first explored for field emission electron microscopy (FEM) and field ion microscopy (FIM) studies [7]. Considering the recipes used for FEM/FIM experiments, Melmed [8] had reported a short review on the experimental conditions for etching $\mathrm{W}$ and Pt/Ir wires for obtaining sharp tips. Changes in the tip geometry were mainly related to the nature and concentration of the etching solution together with the waveform of applied voltage. The influence of the electrochemical cell geometry onto the tip shape was also studied. For example, the double lamellae drop-off etching technique was used for making a 
sharp $\mathrm{W}$ tip from a wire attached to the prong of a tuning fork $[9,10]$. Tips with a curvature radius of $50 \mathrm{~nm}$ were obtained.

Besides efforts to select the best geometry of the cell and etchant solutions, the electric conditions were also adjusted for obtaining sharpest tips. For example, AC polishing process has been improved for Mo tips by controlling the applying voltage, the frequency, the waveform and the bursts [11]. For W tips, Ibe et al. [12] have evidenced that, in addition to the electrochemical conditions, other parameters as the cut-off delays and the wire immersion length modify the tip curvature radius. Shortening the cut-off delay of the etching current after the material wire drops off at the airelectrolyte interface was also found to be critical for the radius of W tip apex [13]. Fotino had proposed an original design for the cell called reverse electrochemical etching geometry [14]. In that configuration a hook-shaped wire, partially covered by an insulating layer except for the free end, allows the formation of a tip from which the bubble flow moves away. In this reverse etching the induced bubbles apply shear forces improving the tip sharpness. A process for achieving a reproducible sharp tip apex was identified from a systematic study of the electropolishing process of $W$ tips [15].

However, the well-known presence of an oxide layer covering the Pt/Ir [16-18] and W [19-26] tips requires a cleaning treatment to obtain optimal tunneling current flow. W tips fabricated by electrochemical etching, cleaned by heating and characterized by Fowler-Nordheim (FN) plot and FIM were reported to display a clean apex with 3-15 nm radius of curvature [24,25]. Recently, the apex radii of tungsten based microemitter tips, ranging from 25 to $500 \mathrm{~nm}$, were measured from SEM images and extracting from FN plots of the field emission characteristics [27].

On the other hand, the fabrication of Pt/Ir tips was less intensively studied than the $\mathrm{W}$ ones, although it is established that platinum based tips are chemically inert and less prone to the oxide layer problem than tungsten. However, platinum-based tips require more complicated fabrication protocols than the $\mathrm{W}$ ones. For example, two stages of electrochemical etching with two different solutions were need to obtain reasonable sharpness for high resolution STM imaging as shown by Libioulle et al. [17] who used $\mathrm{CaCl}_{2}$ electrochemical solution followed by an electrochemical etching in $\mathrm{H}_{2} \mathrm{SO}_{4}$ for sharpening tips in safer conditions than the highly toxic cyanide etchant. Lindhal et al. had presented a method to fabricate sharp Pt/Ir tips which consists of three electrochemical etching steps in two kinds of solution [18]. In 1999, A. H. Sørensen et al. [28] reported a detailed study of the Pt/Ir tip fabrication in two steps. Considering the influence of bubbles on the tip geometry during the continuous etching process with a $50 \mathrm{~Hz}$ voltage, the authors have modulated the voltage applied to the tip with time spaced bursts for a fine etching of the tip (the second etching step). As the delayed voltage bursts allow the far away diffusion of chloride ions insulating layer around the Pt/Ir shank between each pause [28,29]. Later Pt/Ir tips etched from a " $U$ " shape wire using an inverse geometry combined with a sinusoidal $0.7 \mathrm{~V}$ voltage at $100 \mathrm{~Hz}$ were found to present apex radii around $20 \mathrm{~nm}$ [30].

From this analysis of the state of art of manufacturing ultrasharp tips, $\mathrm{W}$ tips are notably easier to fabricate sharper than Pt based ones but considering the chemical inertness of Pt/Ir when compared to W, tips made from a Pt/Ir wire appear well fitted for mounting on a tuning fork probe for combined STM and AFM operations in an ultra-high vacuum (UHV) environment without the need to install additional tip cleaning facilities in the UHV chamber.

In this paper, we report the optimization of an electrochemical etching process yielding reproducible fabrication of ultra-sharp and conductive Pt/Ir tips. We first describe the geometry of the electrochemical cell and the operating conditions to control the etching process of a Pt/Ir tip using a dedicated electronic device. Afterward the obtained tip geometry is observed by scanning electron microscopy (SEM) and finally characterized by field electron emission. As demonstration of their performances, each etched tip once glued onto a quartz tuning fork operating in qPlus configuration, is used as a scanning probe for high resolution imaging both in STM and AFM regulations (UHV, room temperature) of epitaxial graphene layer on reconstructed $6 \mathrm{H}-\mathrm{SiC}(0001)$ surface.

\section{Experiment}

The STM/AFM probe is based on a quartz tuning fork (Citizen America CFS206, 32,768 Hz) configured in qPlus arrangement (Fig. 1). The preparation of an operational probe equipped with a sharp Pt/Ir tip needs a four step procedure with several characterizations of the tip/tuning fork assembly. In the first step, the tuning fork resonator was mounted on a modified UHV VT-AFM Omicron tripod holder. A prong of the tuning fork is strongly fixed on the macor ceramic part with non-conductive epoxy glue (H77 Epotek, Epoxy Technology, USA) while the electrical contacts were established with silver containing conductive epoxy glue (H21D Epotek, Epoxy Technology, USA). Both glues are UHV compatible and their curing conditions were $120{ }^{\circ} \mathrm{C}$ during $1 \mathrm{~h}$. At this stage, the measured value of the quality factor for the tuning fork oscillating in air is at least 1000 . High quality factor values are mandatory to enhance the detection sensitivity during AFM scanning.

In the second step, a $50 \mu \mathrm{m}$ diameter Pt/Ir wire (Pt90/Ir10, Goodfellow, Cambridge Ltd - Huntingdon), was attached to the top of the free prong of the tuning fork using the H21D conductive epoxy glue.

The third step is devoted to the tip fabrication. First, the fixed Pt/ Ir wire was plunged into a $\mathrm{CaCl}_{2}$ aqueous solution $\left(\mathrm{CaCl}_{2} 5 \mathrm{~g}, \mathrm{H}_{2} \mathrm{O}\right.$ $30 \mathrm{ml}$, acetone $2 \mathrm{ml}$ ) [16,17] supported by a Pt ring with an outer diameter of $2 \mathrm{~mm}$ (Fig. 2) $\left(\mathrm{CaCl}_{2}, 2 \mathrm{H}_{2} \mathrm{O}\right.$ was purchased from NORMAPUR $^{\mathrm{TM}}$ AR VWR international Prolabo (Leuven, Belgium)). The wire length in solution was adjusted with a micrometer precision mechanical screw in the reverse etching geometry suggested by Fotino [31] as illustrated in Fig. 2(A). The advantage of this geometry for the tip formation is that the bubbles escape from the etched wire which may induce beneficial shear forces towards the apex of the etched tip improving its sharpness. In fact, the bubbles slide along the etched cone in contrast with a normal cell geometry in which some of them are trapped by the buoyant force onto surface defects of the tip.

The formation of a sharp tip is highly dependent on the waveform, frequency and amplitude parameters of the applied etching current. Fig. 2(B) depicts the schematics of the developed electronics to control these parameters. Due to that the etching rate of platinum increases for decreasing frequency below $50 \mathrm{~Hz}$ [29], the etching signal is based on a burst sinusoidal waveform of $100 \mathrm{~ms}$,

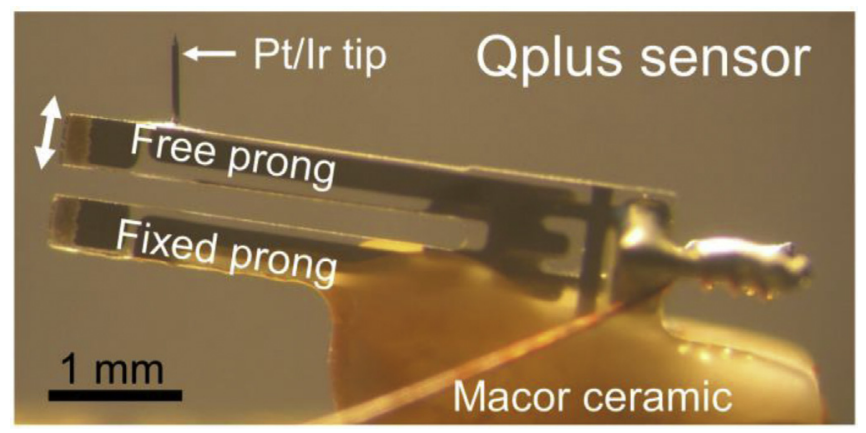

Fig. 1. Home-made qPlus sensor. One prong of a tuning fork is fixed to a macor ceramic plate, the other free prong has a Pt/Ir tip attached to it at the very end. 

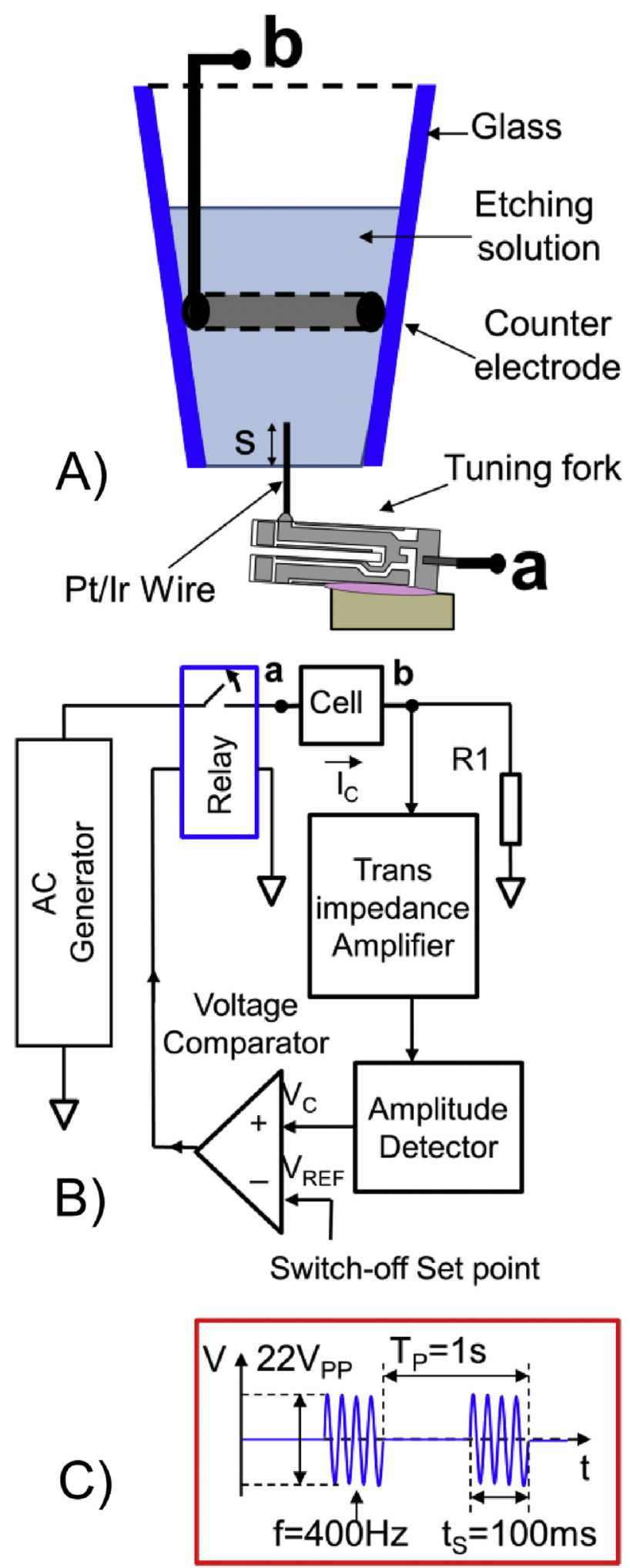

Fig. 2. Schematic of the reverse electrochemical etching set-up for ultra-sharp tip fabrication from Pt/Ir wire. (A) Reverse electrochemical cell with a Pt/Ir ring as a counter electrode (wire diameter $0.25 \mathrm{~mm}$, outer ring diameter $\sim 2 \mathrm{~mm}$ ). The immersion length of the Pt/Ir wire is critical ( $\mathrm{a}$ and $\mathrm{b}$ wires correspond to connections with the electronic device). (B) Schematic diagram of electronic system for controlling the etching process. A sinusoidal voltage trigged by $100 \mathrm{~ms}$ pulses of $1 \mathrm{~s}$ period is amplified by an audio power amplifier to generate a burst signal ( $400 \mathrm{~Hz}, 22 \mathrm{Vp}-\mathrm{p})$. The etching current 'I' circulating in the cell is converted into a voltage signal. Then a voltage comparator controls a relay for stopping the etching process when the etching current 'I' is less than the reference setpoint 'I $\mathrm{I}_{\text {ref }} \alpha \mathrm{V}_{\text {reff. }}$ (C) Waveform of the signal applied on the etching cell to etch Pt/Ir tips. with a carrier frequency of $400 \mathrm{~Hz}$, and triggered by a pulsed signal of a period of $1 \mathrm{~s}$, as shown on Fig. 2(C). Thus, the bubbles and platinum complexes formed during the etching process are removed in the pauses [28] and the solution concentration stays constant along the tip surface. This modulated voltage applied to the electrochemical cell generates the etching current 'I' whose intensity converted into voltage by the transimpedance amplifier is compared to a reference signal (' $\mathrm{V}_{\text {ref }}$ ', setpoint defined by the user) through a voltage comparator. Then, the output of the comparator controls the switching of a relay, opening the circuit and stopping the etching when the etching current becomes smaller than the setpoint ' $V_{\text {ref }}$ '. This automatic switch-off system improves the reproducibility of the tip shape.

At the end of the process, the tip was immersed by $0.25 \mathrm{~mm}$ into a fresh etchant solution. One pulse of the etching voltage (22 Vp-p ac $400 \mathrm{~Hz}$ ) was applied manually in order to remove the possible presence of a contamination layer on the immersed $\mathrm{Pt} / \mathrm{Ir}$ tip as observed in previous studies [28]. We noted that during the tips fabrication process the manufacturing reproducibility is affected by mechanics vibrations of the work-table and for solve this problem, all electrochemical system was supported on an anti-vibration table.

Next, the etched tips are thoroughly washed with hot Milli-Q water bath at $80^{\circ} \mathrm{C}$, then by an ethanol flux and dried in an oven at $120^{\circ} \mathrm{C}$ for $10 \mathrm{~min}$. Then, some tips are observed by SEM (JEOL JSM-5510 LV, resolution limit of $10 \mathrm{~nm}$ ). All of them are finally characterized by field electron emission to obtain FN plots. Such a plot is a convenient and well established method to characterize the mean radius of curvature of the tip apex in which the intensity of the electron emission current (I) from the studied tip is measured as a function of the applied voltage $(\mathrm{V})$ onto a counter electrode. In the FN regime, under the approximation of Spindt et al. [32], I varies as shown by the equation [33]:

$I=A\left(F^{2} / \phi\right) \exp \left(9.84 / \phi^{1 / 2}\right) \exp \left(-6.49 \times 10^{9} \phi^{3 / 2} / F\right)$

with I the current intensity (A), $A$ is a constant, $\varphi$ (the local work function of the material of the tip) in $\mathrm{eV}(\varphi=5.7 \mathrm{eV}$ for Pt/Ir [34]), $F$ is the local electric field related to the applied voltage $V$ via $F=\beta V$ with $\beta=1 / k R$ being the field conversion factor, $\mathrm{R}$ is the curvature radius of the tip apex and $k$ is the field reduction factor that depends on the exact tip geometry and the distance between the tip apex and the counter electrode [7,35]. However, k can reach values between 3 and 35 as reported for tungsten tips [7,13,20-22,24-26]. Dividing the equation (1) by $V^{2}$ and taking the nature logarithm on both sides, we obtain:

$$
\begin{aligned}
\operatorname{Ln}\left(I / V^{2}\right)= & \operatorname{Ln}\left(A \beta^{2} / \phi\right)+9.84 / \phi^{1 / 2} \\
& -\left(6.49 \times 10^{9} \phi^{3 / 2} / \beta\right)(1 / V)
\end{aligned}
$$

From the slope ' $m$ ' of the linear variation of the FN plot $\operatorname{Ln}\left(I / V^{2}\right)$ vs $1 / V$, we can obtain:

$k R=-m / 6.49 \times 10^{9} \phi^{3 / 2}$

with a model of the tip geometry for estimating $\mathrm{k}$, the value of $\mathrm{R}$ can be deduced from the FN plots.

Our combined UHV STM/AFM system was previously described in [3]. Briefly, it consists of a modified VT Omicron microscope coupled to a homemade dual preamplifier connected to a NANONIS-SPECS controller. The oscillation of the tip fixed to a qPlus probe was set to typically 1 to 2 ångströms amplitude with a resonance frequency falling to $30 \mathrm{kHz}$ due to the added mass of the 
glued tip. The scanning probe microscope was driven following three modes of distance regulation. First, the $Z$ distance between the non-oscillating tip and the sample is regulated via the intensity of the tunneling current (Static STM mode or standard STM operation). Second, in dynamic STM mode, the $\mathrm{Z}$ distance regulation is performed via the mean intensity of the tunneling current established between the oscillating tip and the sample. Dynamic STM images are obtained simultaneously with maps of the variation of the frequency shift sensitive to the force gradients between the tip end and the surface. Third, in frequency modulated AFM (FM-AFM) mode, the $Z$ distance regulation is realized using the resonance frequency shift of the oscillating probe. FM-AFM topography images are obtained simultaneously with maps of the mean tunneling current intensity. WSxM [36] or Gwyddion [37] software have been used for the image processing.

The $6 \mathrm{H}-\mathrm{SiC}(0001)$ samples from NovaSiC were first ex situ chemically cleaned including trichlorethylene, acetone, and methanol in an ultrasonic bath. Then the sample was immediately introduced into UHV and treated in situ by resistive heating. After degassing for $8 \mathrm{~h}$ at $600{ }^{\circ} \mathrm{C}$, the sample was annealed for $5 \mathrm{~min}$ at different temperatures between $800{ }^{\circ} \mathrm{C}$ and $1300{ }^{\circ} \mathrm{C}$ by steps of $50^{\circ} \mathrm{C}$ at a base pressure lower than $10^{-9}$ mbar [38]. The samples of epitaxial graphene grown on the $\mathrm{SiC}(0001)$ surface were transferred in air to the STM/AFM chamber. Once in the UHV chamber $\left(5 \times 10^{-10} \mathrm{mbar}\right)$ of the AFM/STM microscope, the samples were heated at $500-550{ }^{\circ} \mathrm{C}$ (Pyrometer Raytek) for $1 \mathrm{~h}$ to remove contamination induced by atmosphere exposition and cooled for $2 \mathrm{~h}$ down to room temperature before AFM/STM observations.

\section{Results and discussion}

\subsection{Fabrication of sharp and clean Pt/Ir tips}

In a first attempt, tips with a sharp apex were obtained by electrochemical etching of a $0.5 \mathrm{~mm}$ immersed wire in a fresh $\mathrm{CaCl}_{2}$ electrolytic solution in the reverse configuration shown in Fig. 2(A). However, the tip shapes present a rather large dispersion of the apex radius values as revealed by SEM observations (not shown here) for a sinusoidal voltage of $22 \mathrm{Vp}-\mathrm{p}$ at $400 \mathrm{~Hz}$ applied on the $\mathrm{Pt} / \mathrm{Ir}$ wire up to the end of the etching process. In the second approach, the sinusoidal voltage of $22 \mathrm{Vp}-\mathrm{p}$ at $400 \mathrm{~Hz}$ was triggered with $100 \mathrm{~ms}$ duration with $1 \mathrm{~Hz}$ repeating frequency (Fig. 2(C)). Furthermore, the etching process was switched off when the mean etching current decreased below a preset threshold defined by the user as a setpoint (Fig. 2(B)). As previously pointed out [28], a periodic burst etching signal canceled the screening effect of the bubble flux on the etching current and favors dilution of the Pt compounds during pause.

The sharpest tips were obtained with the etching switch-off threshold set at $20 \mathrm{~mA}$. A typical time variation of the amplitude of the etching current intensity is reported in Fig. 3.

Fig. 4 shows SEM pictures of typical Pt/Ir tips obtained in these conditions. The surface of the cone forming the tips appears smooth and the curvature radii of the tip apex are around $23 \pm 5 \mathrm{~nm}$.

Several tips prepared following this procedure were characterized in the field emission chamber for estimating the tip radius curvature from Fowler-Nordheim plots as depicted on Fig. 5.

We noticed that the onset of measured emission current is $\sim 1 \mathrm{nA}$. Furthermore we pay attention to limit the current to a few $\mu \mathrm{A}$ in order to prevent any tip heating which would yield blunt tips. Deduced from the slope of the linear part of Fowler-Nordheim plots, we obtain $\mathrm{kR}$ values around $48 \mathrm{~nm}$ with ones at 45 and $34 \mathrm{~nm}$. On the other hand, Schirmeisen et al. [20] report a kR value of $119.5 \mathrm{~nm}$ from a Fowler-Nordheim plot and for a polycrystalline $\mathrm{W}$ tip. Years later, a series of $\mathrm{W}$ tips, fabricated by Lucier et al.

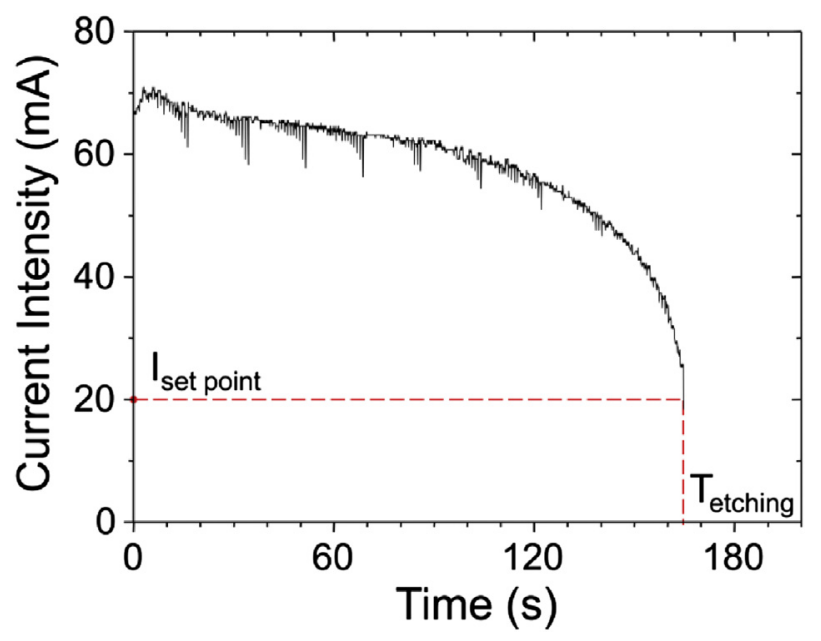

Fig. 3. Time evolution of the amplitude of the etching current.
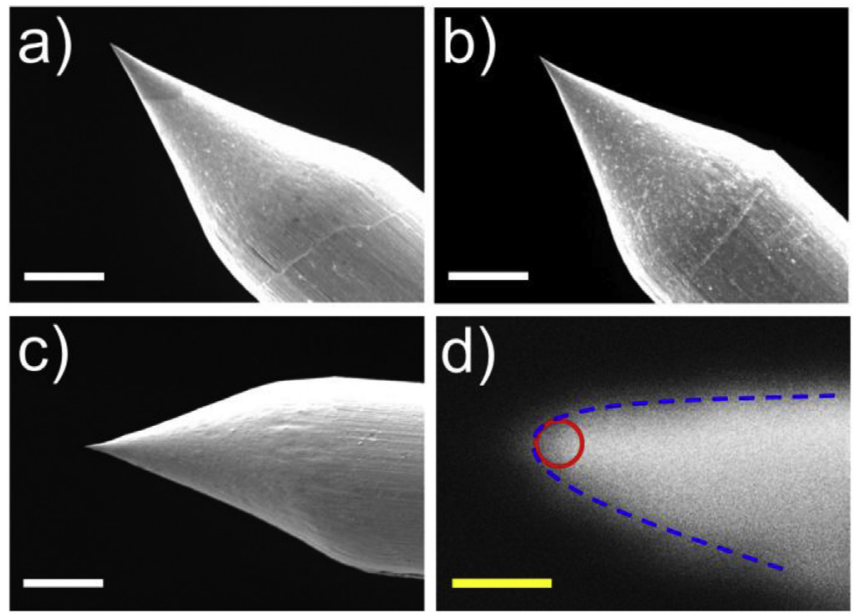

Fig. 4. Scanning Electron Microscopy micrographs of 3 etched Pt/Ir tips with the same electrochemical conditions. Images (a), (b) and (c) display the shape of the tips at low magnification (white scale bar represents 10 micrometers); (d) show the apex of the tip respectively (yellow scale bar represents $100 \mathrm{~nm}$ ). The red circle has a $23 \pm 5 \mathrm{~nm}$ radius. (For interpretation of the references to colour in this figure legend, the reader is referred to the web version of this article.)

[21,22], present kR values between 65 and $137 \mathrm{~nm}$. The SEM images of these tips reveal an apex radius ranging from 20 to $40 \mathrm{~nm}$. Then, Hagedorn et al. [24,25] determined that W (111) tips present an apex radius of $\mathrm{r}<10 \mathrm{~nm}$ from FIM observations while the measured $\mathrm{kR}$ value varies between 122 and $248 \mathrm{~nm}$. From these investigations we verified that the characterization for Pt/Ir tips from Fowler-Nordheim plots has relatively been less investigated [39] than one for W tips [20-22,24,25,27]. We show that our fabricated Pt/Ir tips present kR values lower than those obtained by Schirmeisen et al. [20], Lucier et al. [21,22], and Hagedorn et al. [24,25], reported for sharp W tips.

However, for estimate the apex radius of our fabricated tips its necessary know the $\mathrm{k}$ value, which depends on the tip shape and the distance ' $d$ ' between the tip apex and the counter electrode [7,35]. Values of $\mathrm{k} \sim 5$ is adequate for most geometries encountered in practice [7,35]. Nakamura et al. [13] use a k value of 5 for to estimate the curvature radius of theirs fabricated $\mathrm{W}$ tips experimentally. Schirmeissen et al. [20] find that $\mathrm{k}$ reaches values as high as 35 for W tips with an apex radius $\sim 2.5 \mathrm{~nm}$ and a value of 18 for ones with an apex radius between 5 and $7 \mathrm{~nm}$. More recently, 


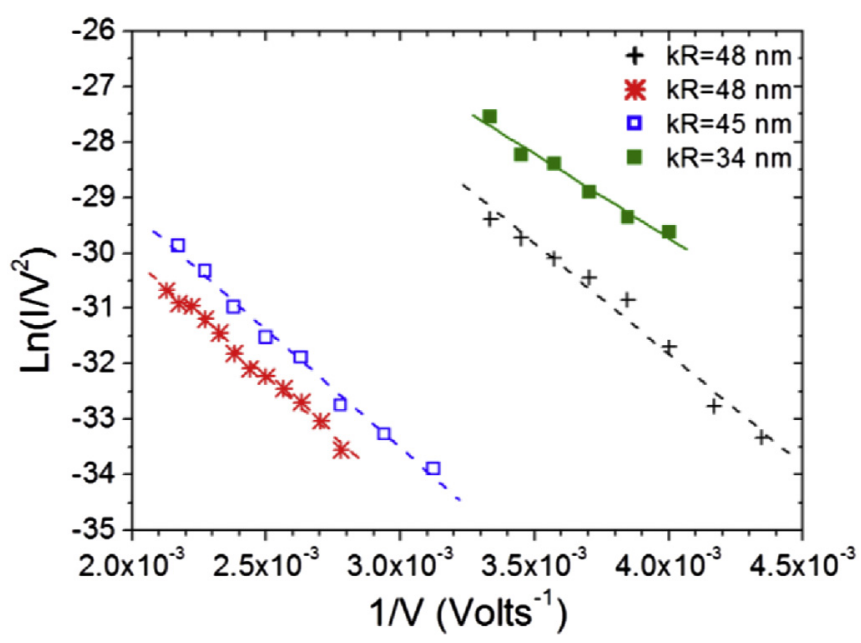

Fig. 5. Fowler-Nordheim plots of four Pt/Ir tips. The slopes of curve, proportional to the product $k R$, exhibit a small dispersion. With $k=5.6$, the tip radius ranges from $6 \mathrm{~nm}$ to $9 \mathrm{~nm}$.

Hagedorn et al. [24,25] assuming a maxima $\mathrm{k}$ factor of 35 for to determine the minimum apex radius of their $\mathrm{W}$ tips manufactured. Lucier et al. [21,22] obtained $\mathrm{k}$ values of 3.3 and 3.4 deduced from the tip radius determined SEM images and the $\mathrm{kR}$ values obtained from Fowler-Nordheim plots.

In this work, the tip apex can be modeled as a paraboloid of revolution [35] in agreement with Fig. 4(d) and the expression for $\mathrm{k}$ is: $k=0.5 \operatorname{Ln}(d / R)$, where $\mathrm{R}$ is the curvature radius of the tip. With

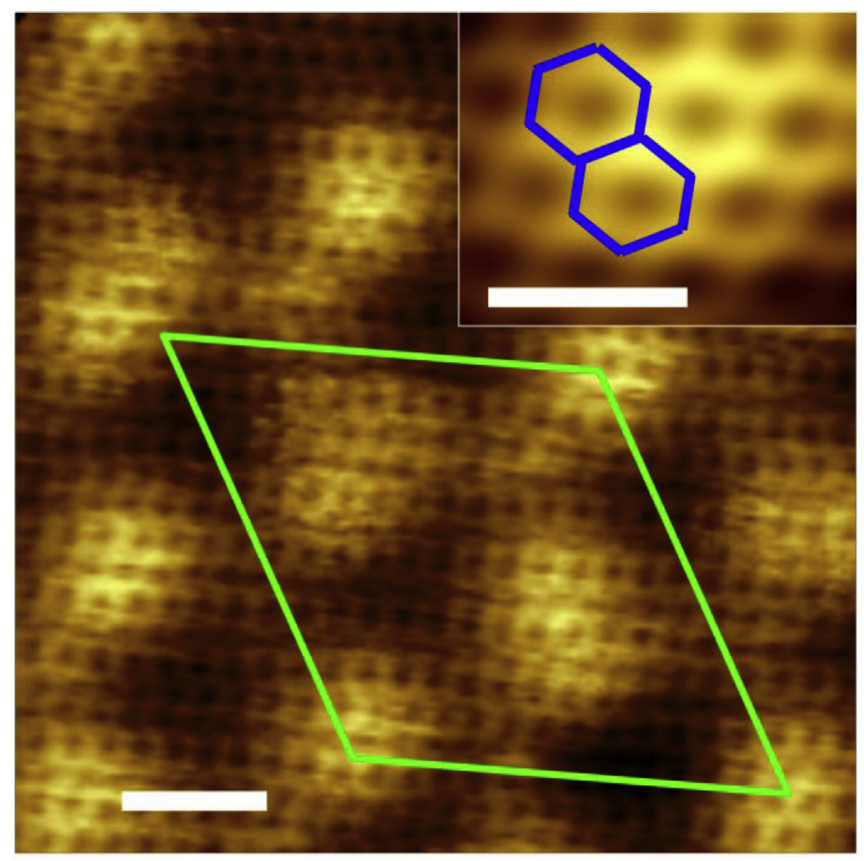

Fig. 6. Constant current STM images obtained with a non-oscillating tip showing the coexistence of two hexagonal lattices of the epitaxial graphene layer on reconstructed $6 \mathrm{H}-\mathrm{SiC}(0001)$ surface: the large yellow spots correspond to the well-known $(6 \sqrt{3} \times 6 \sqrt{3}) \mathrm{R} 30^{\circ}$ lattice of the reconstructed SiC surface (green mesh) and the periodic arrangement of small brown spots to the graphene. The inset displays the atomic lattice of the graphene highlighted by the blue hexagons. $\mathrm{I}_{\mathrm{T}}=300 \mathrm{pA}$, $\mathrm{V}_{\text {sample }}=-50 \mathrm{mV}$. The $\mathrm{Z}$ scale is $71 \mathrm{pm}$ for both images. Scale bars: $1 \mathrm{~nm}$ and $0.5 \mathrm{~nm}$ in the inset. The data were filtered by Fast Fourier Transform in the inset. (For interpretation of the references to colour in this figure legend, the reader is referred to the web version of this article.) the value $k R=\sigma$ known, we resolve the nonlinear equation to obtain an estimate value for ' $k$ ':

$k-(\sigma / d) \exp (2 k)=0$

with $\mathrm{d}=0.5 \mathrm{~mm}$ and $\mathrm{kR}$ values of $34,45,48$ and 48 , we obtain $k$ values between 5.5 and 5.7 smaller than those reported by other authors [24,25] that using a high $k$ value to expect a small value of $\mathrm{R}$. We verified that the $k$ values obtained are close to $k=5$ reported by $[7,13,35]$. From the measured $k R$ values in Fig. 5 and an average $\mathrm{k}$ value of 5.6 yields to $6 \mathrm{~nm}<\mathrm{R}<9 \mathrm{~nm}$, a tip radius smaller than the measured ones in the SEM micrograph in Fig. 4(d), with limited resolution, and ones extracted from SEM images by Lucier et al. [21,22] and Mousa et al. [27].

\subsection{STM/AFM images for testing the tip geometry}

In order to characterize the sharpness and conductivity of the $\mathrm{Pt} / \mathrm{Ir}$ tip apex, normal STM, dynamic STM and FM-AFM images were recorded on a $6 \mathrm{H}-\mathrm{SiC}(0001)$ surface covered by a graphene layer. Fig. 6 presents constant current STM images obtained with a Pt/Ir tip attached on a non-oscillating qPlus probe.

Two lattices can be recognized. The first one with a period of $3.2 \mathrm{~nm}$ corresponds to the well-known $(6 \sqrt{3} \times 6 \sqrt{3}) \mathrm{R} 30^{\circ}$ reconstruction of the buffer layer on a $6 \mathrm{H}-\mathrm{SiC}(0001)$ surface [40-42]. The other lattice formed by hexagons with a period between neighboring centers of $0.24 \mathrm{~nm}$ as shown in the inset is assigned to the epitaxial graphene layer covering the reconstructed SiC surface. As previously reported [40] the relationship between the two
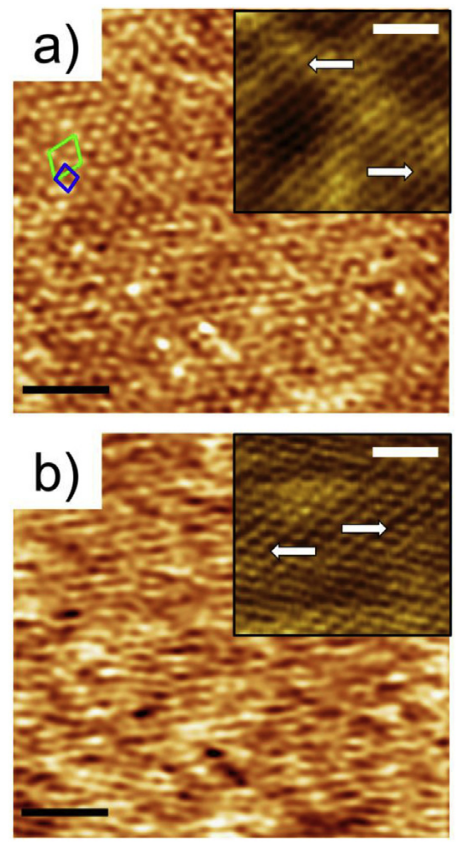

Fig. 7. Dynamic STM topography (a) and map of frequency shift $\Delta \mathrm{f}$ (b) simultaneously recorded on the epitaxial graphene layer on the $(6 \sqrt{3} \times 6 \sqrt{3}) \mathrm{R}^{\circ} 0^{\circ}$ reconstruction of the $6 \mathrm{H}-\mathrm{SiC}(0001)$ surface. $(6 \sqrt{3} \times 6 \sqrt{3}) \mathrm{R} 30^{\circ}$ unit cell (green) and $(6 \times 6)$ quasi-cell (blue) are indicated by diamonds. We noticed that $\Delta \mathrm{f}$ map, proportional to the force gradient, is positive meaning that the tip/surface interaction is mainly repulsive. $\left(\mathrm{V}_{\text {sample }}=-200 \mathrm{mV},\left\langle\mathrm{I}_{\mathrm{T}}>=12 \mathrm{pA}\right.\right.$, oscillation amplitude $\left.=0.12 \mathrm{~nm}\right) . \mathrm{Z}$ scales: (a) 0-60 pm, (b) 27-33 Hz. In the insets are presented the corresponding high resolution images showing in (a) the graphene atomic lattice obtained in dynamic STM mode and in (b) the $\Delta \mathrm{f}$ map modulations due to the graphene lattice. In both insets, the arrows show the graphene lattice (period $0.24 \mathrm{~nm}$ ). Images in the insets were FFT filtered and corrected from drift. Black and white scale bars represent $10 \mathrm{~nm}$ and $1 \mathrm{~nm}$ respectively. (For interpretation of the references to colour in this figure legend, the reader is referred to the web version of this article.) 
lattices can be verified since 13 periods of graphene fit one period of the reconstructed $\mathrm{SiC}(0001)$ surface. These high-resolution STM images which are very similar to results obtained with $\mathrm{W}$ [41] and Pt/Ir [40] tips, clearly demonstrate the high quality of our Pt/Ir tips.

Next, we switched to dynamic STM mode maintaining constant the oscillation amplitude of the probe. The $\mathrm{Z}$ distance regulation of the microscope was now driven by the mean value of the tunneling current $\left\langle\mathrm{I}_{\mathrm{T}}>\right.$. In this operating mode, dynamic STM images of graphene on the SiC surface were obtained with maps of the variation of the frequency shift of the probe. Both images show the SiC reconstruction lattice as illustrated in Fig. 7.

The lattice with a $0.24 \mathrm{~nm}$ period, clearly observed on the dynamic STM images shown in the inset of Fig. 7(a), indicates the presence of the graphene layer. It is worth pointing out that the same lattice is evidenced in the map of frequency shift. As a consequence, Fig. 7(b) and the inset demonstrate that the tip/surface interaction is modulated with $1.9 \mathrm{~nm}$ and $0.24 \mathrm{~nm}$ periods when the tip scans in STM mode the graphene layer in epitaxy on the $6 \mathrm{H}-\mathrm{SiC}(0001)(6 \sqrt{3} \times 6 \sqrt{3}) \mathrm{R} 30^{\circ}$ reconstructed surface. The lattice with a $1.9 \mathrm{~nm}$ periodicity is attributed to the $(6 \times 6)$ quasicell of the underlying reconstructed SiC surface.

Finally, we switched to FM-AFM mode with distance regulation performed with the resonance frequency shift signal. Both the topography at constant resonance frequency shift and the map of variation of the mean tunneling current intensity exhibit a lattice with a $1.9 \mathrm{~nm}$ period which is identified with the $(6 \times 6)$ reconstruction of the $6 \mathrm{H}-\mathrm{SiC}(0001)$ surface. To the best of our knowledge, we emphasize that the images in Fig. 8 are the first showing AFM topography, simultaneously recorded with a mean tunneling current map, of an epitaxial graphene layer on reconstructed $\mathrm{SiC}(0001)$ surface obtained at room temperature and showing the
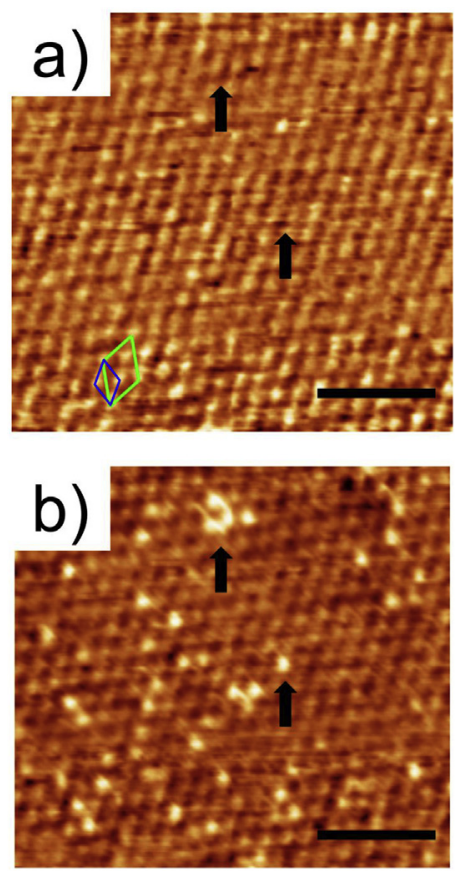

Fig. 8. FM-AFM topography (a) and map of the mean tunneling current (b) simultaneously recorded on an epitaxial graphene layer on the $(6 \sqrt{3} \times 6 \sqrt{3}) \mathrm{R} 30^{\circ}$ reconstruction of the $6 \mathrm{H}-\mathrm{SiC}(0001)$ surface. $(6 \sqrt{3} \times 6 \sqrt{3}) \mathrm{R} 30^{\circ}$ unit cell (green) and $(6 \times 6)$ quasi-cell (blue) are indicated by diamonds. The arrows show protrusions in the mean tunneling current map (b) which are not related to feature in (a). $\Delta \mathrm{f}=+15 \mathrm{~Hz}$. Oscillation amplitude $0.12 \mathrm{~nm}, \mathrm{~V}_{\text {sample }}=-5 \mathrm{mV}$, scale bar $=10 \mathrm{~nm}$. Z scales in (a) $187 \mathrm{pm}$ and in (b) 25.4-41.5 nA. Data filtered by a Gaussian smoothing. (For interpretation of the references to colour in this figure legend, the reader is referred to the web version of this article.)
$(6 \sqrt{3} \times 6 \sqrt{3}) \mathrm{R} 30^{\circ}$ reconstruction structure. This AFM topography obtained in repulsive mode is similar to the one reported by Filleter and Benewitz and for which the tip/surface interaction was not mentioned [43]. Interestingly, the mean tunneling current map exhibits some bright spots probably related to local variations of the density of states near the Fermi level which are not related to features in the corresponding AFM topography. This will be discussed in a forthcoming paper [44].

\section{Conclusions}

Sharp and conductive Pt/Ir tips have been reproducibly fabricated by a one stage electrochemical etching process in $\mathrm{CaCl}_{2}$ solution using an inverse geometry of the electrochemical cell and a dedicated electronic device which allows us to control sequences of alternative voltages. In these conditions, tips with a mean apex radius smaller than $10 \mathrm{~nm}$ were routinely produced as demonstrated by electron field emission measurements through Fowler-Nordheim plots. As probes for a combined STM/AFM system these tips mounted on a quartz tuning fork working in a qPlus configuration allow us to achieve high resolution images of the epitaxial graphene layer on $(6 \sqrt{3} \times 6 \sqrt{3}) \mathrm{R}^{\circ} 0^{\circ}$ reconstruction of the $6 \mathrm{H}-\mathrm{SiC}(0001)$. The surface structure was explored at room temperature by normal STM, dynamic STM and for the first time by FM-AFM. High resolution images of the graphene lattice were achieved in STM mode, dynamic STM mode together with maps of frequency shift. We are working on the controlled fabrication of sharper tips for which we soon hope to achieve atomic resolution on graphene layer by FM-AFM at room temperature.

\section{Acknowledgments}

We wish to thank Laurent Pham-Van (CEA/IRAMIS/SPEC/LEPO) for fruitful discussions on the tip etching process and Sylvain Foucquart (CEA/IRAMIS/NIMBE/LIONS) for his technical assistance.

José Antonio Morán Meza would also like to acknowledge financial support from the Peru's National Council of Science and Technology (Concytec), the Réseau Thématique de Recherche Avancée (RTRA-Triangle de la Physique), and the CEA/Direction des Relations Internationales (DRI).

\section{References}

[1] F.J. Giessibl, High-speed force sensor for force microscopy and profilometry utilizing a quartz tuning fork, Appl. Phys. Lett. 73 (26) (1998) 3956-3958, http://dx.doi.org/10.1063/1.122948.

[2] L. Gross, F. Mohn, N. Moll, P. Liljeroth, G. Meyer, The chemical structure of a molecule resolved by atomic force microscopy, Science 325 (2009) 1110-1114, http://dx.doi.org/10.1126/science.1176210.

[3] J. Polesel-Maris, C. Lubin, F. Thoyer, J. Cousty, Combined dynamic scanning tunneling microscopy and frequency modulation atomic force microscopy investigations on polythiophene chains on graphite with a tuning fork sensor, J. Appl. Phys. 109 (2011) 074320/10, http://dx.doi.org/10.1063/1.3556437.

[4] E.N. Voloshina, Y.S. Dedkov, S. Torbrügge, A. Thissen, M. Fonin, Graphene on $\mathrm{Rh}(111)$ : scanning tunneling and atomic force microscopies studies, Appl. Phys. Lett. 100 (2012) 241606/4, http://dx.doi.org/10.1063/1.4729549.

[5] L. Pham Van, V. Kyrylyuk, J. Polesel-Maris, F. Thoyer, C. Lubin, J. Cousty, Experimental three-dimensional description of the liquid hexadecane/ graphite interface, Langmuir 25 (2009) 2005-2008, http://dx.doi.org/10.1021/ la803665k.

[6] J. Polesel-Maris, J. Legrand, T. Berthelot, A. Garcia, P. Viel, A. Makky, et al., Force spectroscopy by dynamic atomic force microscopy on bovine serum albumin proteins changing the tip hydrophobicity, with piezoelectric tuning fork selfsensing scanning probe, Sensors Actuators B Chem. 161 (2012) 775-783. http://dx.doi.org/10.1016/j.snb.2011.11.032.

[7] R. Gomer, Field Emission and Field Ionization, Harvard University Press, Cambridge, Mass, 1961. http://physics.unm.edu/courseware/gt/exercise16. files/Field_Emission_and_Field_Ionization.pdf.

[8] A.J. Melmed, The art and science and other aspects of making sharp tips, J. Vac. Sci. Technol. B 9 (2) (1991) 601-608, http://dx.doi.org/10.1116/1.585467.

[9] M. Klein, G. Schwitzgebel, An improved lamellae drop-off technique for sharp 
tip preparation in scanning tunneling microscopy, Rev. Sci. Instrum. 68 (8) (1997) 3099-3103, http://dx.doi.org/10.1063/1.1148249.

[10] M. Kulawik, M. Nowicki, G. Thielsch, L. Cramer, H.-P. Rust, H.-J. Freund, et al., A double lamellae dropoff etching procedure for tungsten tips attached to tuning fork atomic force microscopy/scanning tunneling microscopy sensors, Rev. Sci. Instrum. 74 (2) (2003) 1027-1030, http://dx.doi.org/10.1063/1.1532833.

[11] H. Morikawa, K. Goto, Reproducible sharp-pointed tip preparation for field ion microscopy by controlled ac polishing, Rev. Sci. Instrum. 59 (10) (1988) 2195-2197, http://dx.doi.org/10.1063/1.1139985.

[12] J.P. Ibe, P.P. Bey Jr., S.L. Brandow, R.A. Brizzolara, N.A. Burnham, D.P. DiLella, et al., On the electrochemical etching of tips for scanning tunneling microscopy, J. Vac. Sci. Technol. A 8 (4) (1990) 3570-3575, http://dx.doi.org/10.1116/1.576509.

[13] Y. Nakamura, Y. Mera, K. Maeda, A reproducible method to fabricate atomically sharp tips for scanning tunneling microscopy, Rev. Sci. Instrum. 70 (8) (1999) 3373-3376, http://dx.doi.org/10.1063/1.1149921.

[14] M. Fotino, Tip sharpening by normal and reverse electrochemical etching, Rev. Sci. Instrum. 64 (1) (1993) 159-167, http://dx.doi.org/10.1063/1.1144419.

[15] B.-F. Ju, Y.-L. Chen, M. Fu, Y. Chen, Y. Yang, Systematic study of electropolishing technique for improving the quality and production reproducibility of tungsten STM probe, Sensors Actuators A Phys. 155 (2009) 136-144, http:// dx.doi.org/10.1016/j.sna.2009.08.013.

[16] L. Libioulle, Y. Houbion, J.-M. Gilles, Very sharp gold and platinum tips to modify gold surfaces in scanning tunneling microscopy, J. Vac. Sci. Technol. B 13 (3) (1995) 1325-1331, http://dx.doi.org/10.1116/1.587847.

[17] L. Libiouille, Y. Houbion, J.-M. Gilles, Very sharp platinum tips for scanning tunneling microscopy, Rev. Sci. Instrum. 66 (1) (1995) 97-100, http: dx.doi.org/10.1063/1.1146153.

[18] J. Lindahl, T. Takanen, L. Montelius, Easy and reproducible method for making sharp tips of Pt/Ir, J. Vac. Sci. Technol. B 16 (6) (1998) 3077-3081, http:/ dx.doi.org/10.1116/1.590445.

[19] I. Ekvall, E. Wahlström, D. Claesson, H. Olin, E. Olsson, Preparation and characterization of electrochemically etched W tips for STM, Meas. Sci. Technol. 10 (1999) 11-18, http://dx.doi.org/10.1088/0957-0233/10/1/006.

[20] A. Schirmeisen, Metallic Adhesion and Tunneling at the Atomic Scale, Doctoral dissertation, McGill University, 1999.

[21] A. Lucier, Preparation and Characterization of Tungsten Tips Suitable for Molecular Electronics Studies, Master dissertation, McGill University, 2004, http://www.physics.mcgill.ca/ peter/theses/lucier.pdf.

[22] A.-S. Lucier, H. Mortensen, Y. Sun, P. Grütter, Determination of the atomic structure of scanning probe microscopy tungsten tips by field ion microscopy, Phys. Rev. B 72 (2005) 235420/9, http://dx.doi.org/10.1103/ PhysRevB.72.235420.

[23] Z.Q. Yu, C.M. Wang, Y. Du, S. Thevuthasan, I. Lyubinetsky, Reproducible tip fabrication and cleaning for UHV STM, Ultramicroscopy 108 (9) (2008) 873-877, http://dx.doi.org/10.1016/j.ultramic.2008.02.010.

[24] T. Hagedorn, Atomic Contacts Characterized by Force and Current, Doctoral dissertation, McGill University, 2010.

[25] T. Hagedorn, M. El Ouali, W. Paul, D. Oliver, Y. Miyahara, P. Grütter, Refined tip preparation by electrochemical etching and ultrahigh vacuum treatment to obtain atomically sharp tips for scanning tunneling microscope and atomic force microscope, Rev. Sci. Instrum. 82 (2011) 113903/5, http://dx.doi.org/ 10.1063/1.3660279.

[26] M. Setvín, J. Javorský, D. Turčinková, I. Matolínová, P. Sobotík, P. Kocán, et al., Ultrasharp tungsten tips-characterization and nondestructive cleaning Ultramicroscopy 113 (2012) 152-157, http://dx.doi.org/10.1016/ j.ultramic.2011.10.005.
[27] K.O. Mussa, M.S. Mousa, A. Fischer, Information extraction from FN plots of tungsten microemitters, Ultramicroscopy 132 (2013) 48-63, http:// dx.doi.org/10.1016/j.ultramic.2013.02.023.

[28] A.H. Sørensen, U. Hvid, M.W. Mortensen, K.A. Mørch, Preparation of platinumiridium scanning probe microscopy tips, Rev. Sci. Instrum. 70 (7) (1999) 3059-3067, http://dx.doi.org/10.1063/1.1149891.

[29] J. Llopis, A. Sancho, Electrochemical corrosion of platinum in hydrochloric acid solutions, J. Electrochem. Soc. 108 (8) (1961) 720-726, http://dx.doi.org/ $10.1149 / 1.2428205$.

[30] I. V Borzenets, I. Yoon, M.M. Prior, B.R. Donald, R.D. Mooney, G. Finkelstein, Ultra-sharp metal and nanotube-based probes for applications in scanning microscopy and neural recording, J. Appl. Phys. 111 (2012) 074703/6, http:// dx.doi.org/10.1063/1.3702802.

[31] M. Fotino, Nanotips by reverse electrochemical etching, Appl. Phys. Lett. 60 (23) (1992) 2935-2937, http://dx.doi.org/10.1063/1.107468.

[32] C.A. Spindt, I. Brodie, L. Humphrey, E.R. Westerberg, Physical properties of thinfilm field emission cathodes with molybdenum cones, J. Appl. Phys. 47 (1976) 5248-5263, http://dx.doi.org/10.1063/1.322600.

[33] M.S. Wang, J.Y. Wang, L.-M. Peng, Engineering the cap structure of individual carbon nanotubes and corresponding electron field emission characteristics, Appl. Phys. Lett. 88 (2006) 243108, http://dx.doi.org/10.1063/1.2208941.

[34] J. Chen, Introduction to Scanning Tunneling Microscopy, Oxford University Press, New York, 1993.

[35] W.P. Dyke, W.W. Dolan, Field emission, Adv. Electron. Electron Phys. 8 (1956) 89-185, http://dx.doi.org/10.1016/S0065-2539(08)61226-3.

[36] I. Horcas, R. Fernández, J.M. Gómez-Rodríguez, J. Colchero, J. Gómez-Herrero, A.M. Baro, WSXM: a software for scanning probe microscopy and a tool for nanotechnology, Rev. Sci. Instrum. 78 (2007) 013705, http://dx.doi.org/ 10.1063/1.2432410.

[37] D. Nečas, P. Klapetek, Gwyddion: an open-source software for SPM data analysis, Cent. Eur. J. Phys. 10 (1) (2011) 181-188, http://dx.doi.org/10.2478/ s11534-011-0096-2.

[38] J. Penuelas, A. Ouerghi, D. Lucot, C. David, J. Gierak, Surface morphology and characterization of thin graphene films on SiC vicinal substrate, Phys. Rev. B 79 (2009) 033408/4, http://dx.doi.org/10.1103/PhysRevB.79.033408.

[39] C. Dong, M.C. Gupta, G.R. Myneni, Influences of Surface Adsorption on Field Emission Perfomances for W, Pt/Ir and Multi-wall Carbon Nanotube Emitters, 2002. https://www.jlab.org/div_dept/admin/publications/papers/02/ACP0201.pdf.

[40] F. Varchon, P. Mallet, J.-Y. Veuillen, L. Magaud, Ripples in epitaxial graphene on the Si-terminated SiC(0001) surface, Phys. Rev. B 77 (2008) 235412, http:// dx.doi.org/10.1103/PhysRevB.77.235412.

[41] U. Starke, C. Riedl, Epitaxial graphene on SiC (0001) and SiC (000-1): from surface reconstructions to carbon electronics, J. Phys. Condens. Matter 21 (2009) 134016/12, http://dx.doi.org/10.1088/0953-8984/21/13/134016.

[42] A. Ouerghi, M.G. Silly, M. Marangolo, C. Mathieu, M. Eddrief, M. Picher, et al., Large-area and high-quality epitaxial graphene on off-axis SiC wafers, ACS Nano 6 (7) (2012) 6075-6082, http://dx.doi.org/10.1021/nn301152p.

[43] T. Filleter, R. Bennewitz, Structural and frictional properties of graphene films on $\mathrm{SiC}(0001)$ studied by atomic force microscopy, Phys. Rev. B 81 (2010) 155412, http://dx.doi.org/10.1103/PhysRevB.81.155412.

[44] J.A. Morán Meza, C. Lubin, F. Thoyer, J. Cousty, Tip induced mechanical deformation of epitaxial graphene grown on reconstructed $6 \mathrm{H}-\mathrm{SiC}(0001)$ surface during scanning tunneling and atomic force microscopy studies, Nanotechnology 26 (2015) 255704, http://dx.doi.org/10.1088/0957-4484/26/ $25 / 255704$. 